\title{
Synthesis and Application of 1,2-Aminoalcohols with Neoisopulegol-Based Octahydrobenzofuran Core
}

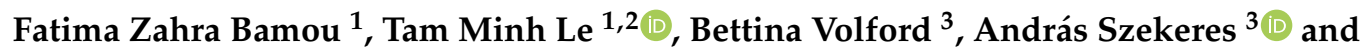 \\ Zsolt Szakonyi ${ }^{1,4, *(\mathbb{C})}$
}

1 Institute of Pharmaceutical Chemistry, University of Szeged, Interdisciplinary excellent center, H-6720 Szeged, Eötvös utca 6, Hungary; fatima.z@pharm.u-szeged.hu (F.Z.B.); leminhtam@pharm.u-szeged.hu (T.M.L.)

2 MTA-SZTE Stereochemistry Research Group, Hungarian Academy of Sciences, H-6720 Szeged, Eötvös utca 6, Hungary

3 Department of Microbiology, University of Szeged, 6726 Szeged, Közép fasor 52, Hungary; bettina.volford86@gmail.com (B.V.); andras.j.szekeres@gmail.com (A.S.)

4 Interdisciplinary Centre of Natural Products, University of Szeged, H-6720 Szeged, Eötvös utca 6, Hungary

* Correspondence: szakonyi@pharm.u-szeged.hu; Tel.: +36-62-546809; Fax: +36-62-545705

Academic Editors: Antal Csámpai, Panayiotis A. Koutentis and Antonio Massa

Received: 22 November 2019; Accepted: 16 December 2019; Published: 19 December 2019

\begin{abstract}
A library of 1,2-aminoalcohol derivatives with a neoisopulegol-based octahydrobenzofuran core was developed and applied as chiral catalysts in the addition of diethylzinc to benzaldehyde. The allylic chlorination of (+)-neoisopulegol, derived from natural (-)-isopulegol followed by cyclization, gave the key methyleneoctahydrobenzofuran intermediate. The stereoselective epoxidation of the key intermediate and subsequent oxirane ring opening with primary amines afforded the required 1,2-aminoalcohols. The ring closure of the secondary amine analogues with formaldehyde provided spiro-oxazolidine ring systems. The dihydroxylation of the methylenetetrahydrofuran moiety with $\mathrm{OsO}_{4} / \mathrm{NMO}$ (4-methylmorpholine $\mathrm{N}$-oxide) resulted in the formation of a neoisopulegol-based diol in a highly stereoselective reaction. The antimicrobial activity of both the aminoalcohol derivatives and the diol was also explored.
\end{abstract}

Keywords: neoisopulegol; octahydrobenzofuran; 1,2-aminoalcohol; chiral catalyst; antimicrobial activity

\section{Introduction}

The benzofuran moiety is prevalent in a great number of biologically active compounds and natural products [1,2]. Moreover, compounds bearing this ring system are also promising key intermediates in the preparation of natural products and clinical medicines [3-5]. Due to the availability of these building blocks, numerous methods have been developed for the preparation of benzofuran systems [6-11]. However, only a few examples of the synthesis of octahydrobenzofuran derivatives have been reported including free-radical reactions [12,13], hydrogenation [14,15], tandem conjugate addition [16], base- [17] or acid-catalyzed cyclization [18], and photochemical rearrangement [19]. Furthermore, octabenzohydrofuran derivatives are well-known versatile precursors for the construction of a variety of therapeutic drugs [20]. For example, $( \pm)$-adunctin B and its modified derivatives that bear a hexahydrobenzofurane moiety have shown antibacterial effects toward Micrococcus luteus [21]. (-)-Siccanin exhibits potent antifungal activity against several pathogenic fungi, and its clinical effectiveness against surface mycosis is also known [22].

The 1,2-aminoalcohol moiety is present in a wide range of compounds that exhibit pharmaceutically and biologically interesting properties [23]. For example, compounds bearing the hydroxyethylamine core have the capacity to inhibit aspartic protease enzymes and are widely used as anti-HIV [24,25], 
antimalarial [26-28], and antileishmanial [29] agents. The 1,2-aminoalcohol function is found in a broad range of $\beta$-adrenergic blockers that are used extensively in the management of cardiovascular disorders [30], including hypertension, angina pectoris and cardiac arrhythmias, and other disorders that are related to the sympathetic nervous system [31,32].

1,2-aminoalcohols have also been demonstrated to be excellent chiral auxiliaries and chiral catalysts in asymmetric synthesis [33]. To achieve new, efficient, and commercially available chiral catalysts, natural chiral terpenes, such as $\alpha$-pinene [34-38], $\beta$-pinene [34,39], (-)-3-carene [39,40], $(-)$-verbenone [41,42], (-)-fenchone [43,44], (+)-camphor [43,45,46], and (-)-menthone [47] have proven to be excellent sources for the synthesis of bifunctional chiral compounds and heterocycles.

In the present work, we set out to create a compound library with a (+)-neoisopulegol-based octahydrobenzofuran core and 1,2-aminoalcohol moieties. The synthesis started from commercially available (-)-isopulegol and then utilizing the resulting 1,2-aminoalcohol derivatives as chiral catalysts in the enantioselective addition of diethylzinc to benzaldehyde. Furthermore, the antimicrobial activities of the synthesized compounds were also tested on multiple bacterial and fungal strains.

\section{Results}

\subsection{Synthesis of Key Intermediate 3}

Key intermediate (-)-3-methylenetetrahydrofuran 3 was prepared from commercially available (-)-isopulegol 1 by oxidizing its hydroxyl function, followed by the stereoselective reduction of the resulting carbonyl group, thus providing (+)-neoisopulegol 2 [48-51]. The allylic chlorination of (+)-neoisopulegol 2 was followed by the cyclization-produced (-)-methylenetetrahydrofuran 3 [52-55], which was transformed into (-)-methylenetetrahydrofuran 4 by allylic oxidation after applying the literature method [55,56] (Figure 1).

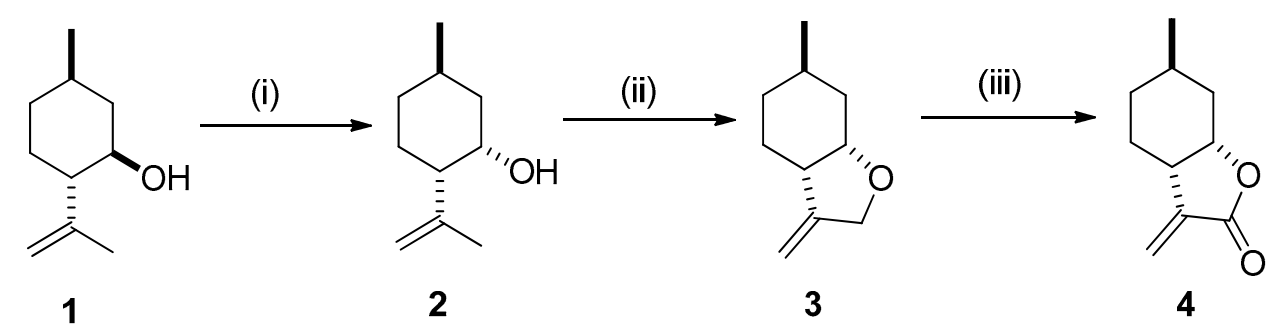

Figure 1. Synthesis of (-)-isopulegol-based methylenetetrahydrofuran 3: (i) PCC (2 equivalents), DCM (Dichloromethane), $25^{\circ} \mathrm{C}, 48 \mathrm{~h}, 80 \%$ than L-selectride (1.5 equivalents) dry THF, $-78^{\circ} \mathrm{C}, 1 \mathrm{~h}, 90 \%$ [48-51];

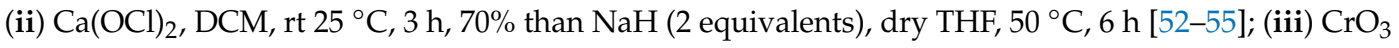
(3 equivalents), DCM/pyridine, reflux, $1.5 \mathrm{~h}, 84 \%$ [55,56].

\subsection{Synthesis of Ispulegol-Based 1,2-Aminoalcohols}

Our previous work has shown that epoxidation with $t-\mathrm{BuOOH}$ in the presence of vanadyl acetylacetonate $\left(\mathrm{VO}(\mathrm{acac})_{2}\right.$ ) as a catalyst can be successfully applied to prepare a new family of neoisopulegol-based chiral aminodiol libraries [57]. However, upon applying this condition with $3,(-)$ - $\alpha$-methylene- $\gamma$-butyrolactone 4 was observed as the major product. The formation of 4 was explained by the allylic oxidation process shown in Figure $2[58,59]$. Finally, the synthesis of epoxide 5 was achieved by reacting 3 with mCPBA (meta-Chloroperoxybenzoic acid) in a stereoselective reaction (Scheme 1) [60-63]. 


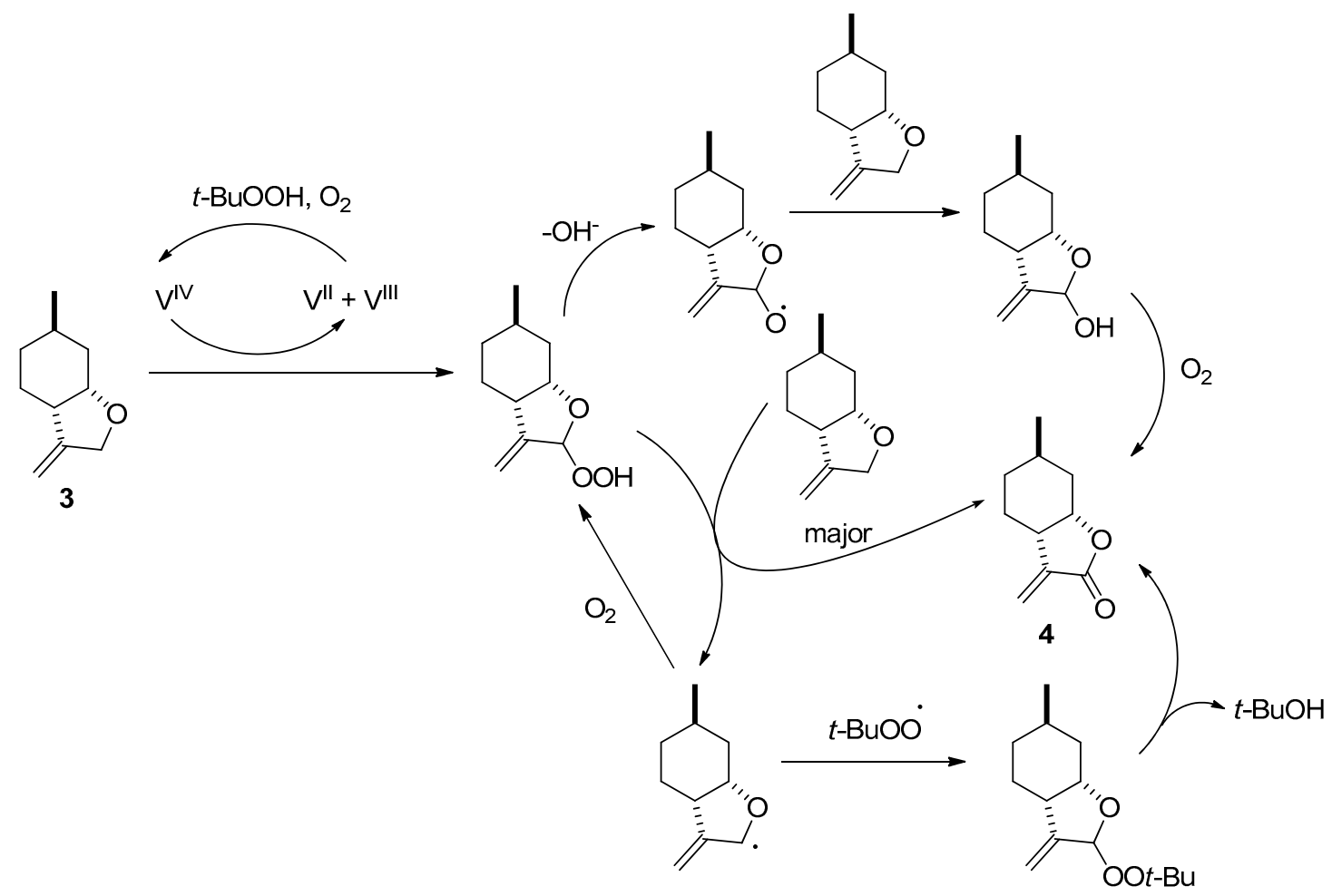

Figure 2. Proposed reaction pathway of allylic oxidation of 3.

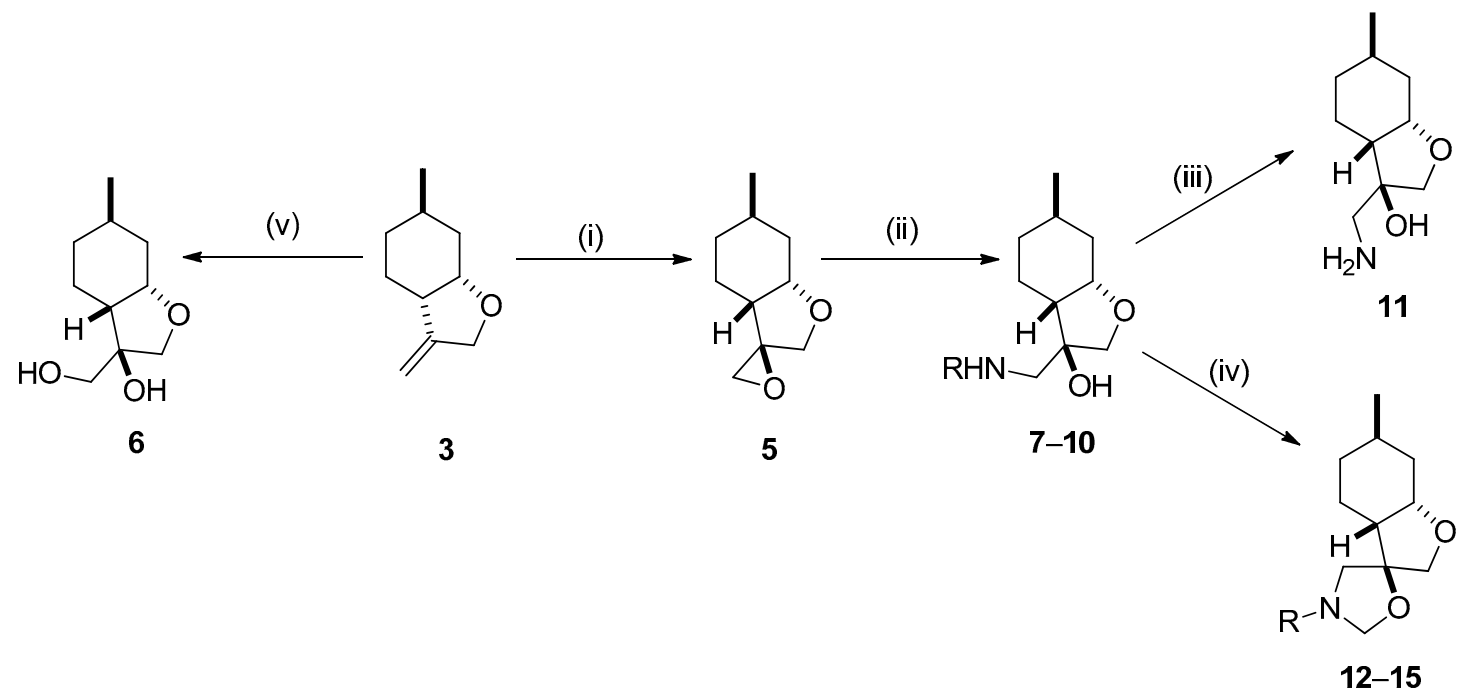

7, 12: $\mathrm{R}=\mathrm{CH}(\mathrm{Me}) \mathrm{Ph}(R) ;$ 8, 13: $\mathrm{R}=\mathrm{CH}(\mathrm{Me}) \mathrm{Ph}(\mathrm{S}) ;$ 9, 14: $\mathrm{R}=\mathrm{CH}_{2} \mathrm{Ph} ; 10,15: \mathrm{R}=\mathrm{CH}(\mathrm{Me})_{2}$

Scheme 1. (i) mCPBA (2 equivalents), $\mathrm{Na}_{2} \mathrm{HPO}_{4} .2 \mathrm{H}_{2} \mathrm{O}$ (3 equivalents), $25^{\circ} \mathrm{C}, 2 \mathrm{~h}, 23 \%$; (ii) $\mathrm{RNH}_{2}(2$ equivalents), $\mathrm{LiClO}_{4}$ (1 equivalent), $\mathrm{MeCN}, 70-80{ }^{\circ} \mathrm{C}, 6 \mathrm{~h}, 65-85 \%$; (iii) $5 \% \mathrm{Pd} / \mathrm{C}, \mathrm{H}_{2}$ ( $1 \mathrm{~atm}$ ), $\mathrm{MeOH}$, $25{ }^{\circ} \mathrm{C}, 24 \mathrm{~h}, 70-75 \%$; (iv) $35 \% \mathrm{HCHO}, \mathrm{Et}_{2} \mathrm{O}, 25{ }^{\circ} \mathrm{C}, 1 \mathrm{~h}, 50-90 \%$; (v) $2 \% \mathrm{OsO}_{4} / \mathrm{t}-\mathrm{BuOH}, 50 \% \mathrm{NMO} / \mathrm{H}_{2} \mathrm{O}$, acetone, $25^{\circ} \mathrm{C}, 24 \mathrm{~h}, 50 \%$.

Given that we clearly demonstrated in previous works $[64,65]$ that the substitution of the nitrogen atom of aminoalcohols definitely influences the efficiency of their catalytic activity, aminoalcohol library 7-10 was prepared through the aminolysis of epoxide $\mathbf{5}$ with primary amines and lithium perchlorate as a catalyst $[66,67]$. Since the ring closure of monoterpene-based aminoalcohols with rigid structures has been shown to enhance their catalytic potential in our earlier experiments $[64,65,68]$, the treatment of aminoalcohols 7-10 with formaldehyde at room temperature resulted in the formation 
of spiro-oxazolidines 12-15. The debenzylation by hydrogenolysis of compounds 7-9 over Pd/C in $\mathrm{MeOH}$ provided primary aminoalcohol 11 in moderate yields (Scheme 1). Since neither the hydrogenolysis of $N$-benzyl analogues 7-10 nor the formation of an oxazolidine ring system by ring closure with formaldehyde had an effect on the absolute configuration of $\mathrm{C}-3$, the relative configuration of the chiral centers of $\mathbf{1 1 - 1 5}$ is known to be the same as that of $\mathbf{7 - 1 0}[64,65,68]$.

The syn-selective dihydroxylation of compound 3 with $\mathrm{OsO}_{4}$ in the presence of a stoichiometric amount of the co-oxidant, NMO (4-methylmorpholine $\mathrm{N}$-oxide) produced product 6 as a single diastereomer in a moderate yield $[68,69]$ (Scheme 1).

The relative stereochemistry of aminoalcohols 7-10 and diol $\mathbf{6}$ was established by coupling constant data and the NOESY (Nuclear Overhauser Effect SpecroscopY) spectral analysis. The large coupling constant of H-9 $\left(J_{4,9}=11.2 \mathrm{~Hz}\right.$ with 6 and $J_{4,9}=12.2 \mathrm{~Hz}$ with 7-10) indicated that it should be axially oriented, while the coupling constant values between H-3 and H-4 $\left(J_{4,3}=J_{3,4}=2.3 \mathrm{~Hz}\right.$ with 6 and $J_{4,3}=J_{3,4}=2.2-3.0 \mathrm{~Hz}$ with 7-10) supported their equatorial orientation. Furthermore, NOESY correlations between OH-7 and $\mathrm{H}-3$ as well as OH-7 and H-4 protons in DMSO- $d 6$ (Dimethylsulfoxide- $d 6$ ) indicated that these groups were oriented in the same direction (see Supporting Information), Therefore, the structures of 6-10 were concluded, as shown on Figure 3. The stereochemistry of $\mathbf{1 1}$ and $\mathbf{1 2 - 1 5}$ was proven in a similar manner by $1 \mathrm{D}$ and $2 \mathrm{D}$ NMR measurements.

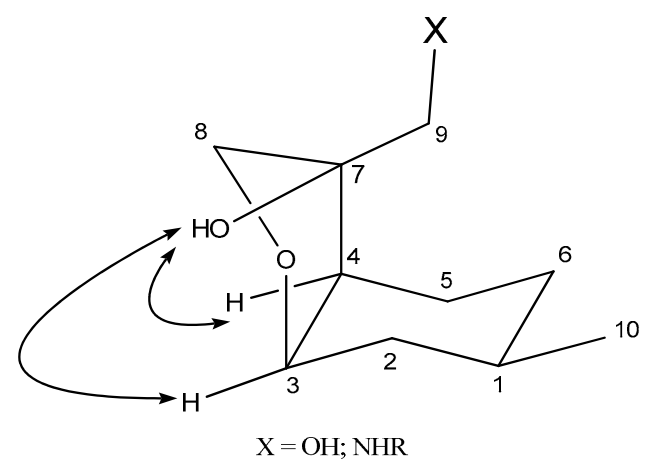

Figure 3. Determination of relative configuration of aminoalcohols 7-10 and diol $\mathbf{6}$.

\subsection{Application of Aminoalcohol Derivatives as Chiral Ligands for Catalytic Addition of Diethylzinc to Benzaldehyde}

Aminoalcohol derivatives 7-15 were applied as chiral catalysts in the enantioselective addition of diethylzinc to benzaldehyde 16 to form (S)- and (R)-1-phenyl-1-propanol 17 (Scheme 2).<smiles>O=Cc1ccccc1</smiles>

16<smiles>CCC(O)c1ccccc1</smiles>

(S)-17<smiles>CCC(O)c1ccccc1</smiles>

$(R)-17$

Scheme 2. Model reaction for enantioselective catalysis.

The enantiomeric purity of 1-phenyl-1-propanols (S)-17 and ( $R$-17 was determined by GC analysis on a Chirasil-DEX CB column using literature methods [70,71]. A low-to-moderate enantioselectivity was observed. Aminoalcohols afforded the (S)-enantiomer (except 7, where a weak $(R)$ selectivity was observed), while the formation of the $(R)$-enantiomer was predominant when spiro-oxazolidines were applied as catalysts (Table 1). Aminoalcohol 8 showed the best catalytic activity $(e e=40 \%$ ) with an (S)-selectivity (entry 2 ). The obtained results clearly indicate that the spiro-oxazolidine ring had a poorer catalytic performance, probably due to the flexible spiro system. These results are in good correlation with those observed with pinane- or sabinane-based spiro-oxazolidines in our earlier studies [72,73]. 
Table 1. Addition of diethylzinc to benzaldehyde catalyzed by aminoalcohol derivatives.

\begin{tabular}{ccccc}
\hline Entry & Ligand & Yield $^{\mathbf{a}} \mathbf{( \% )}$ & $\boldsymbol{e e}^{\mathbf{b}} \mathbf{( \% )}$ & Configuration of the Major Product $^{\mathbf{c}}$ \\
\hline 1 & $\mathbf{7}$ & 86 & 8 & $(R)$ \\
2 & $\mathbf{8}$ & 90 & 40 & $(S)$ \\
3 & $\mathbf{9}$ & 89 & 11 & $(S)$ \\
4 & $\mathbf{1 0}$ & 93 & 4 & $(S)$ \\
5 & $\mathbf{1 1}$ & 95 & 7 & $(S)$ \\
6 & $\mathbf{1 2}$ & 88 & 11 & $(R)$ \\
7 & $\mathbf{1 3}$ & 90 & 3 & $(R)$ \\
8 & $\mathbf{1 4}$ & 82 & 6 & $(R)$ \\
9 & $\mathbf{1 5}$ & 80 & 7 & $(R)$
\end{tabular}

a After silica column chromatography. ${ }^{b}$ Determined using the crude product by GC analysis (Chirasil-DEX CB column). ${ }^{c}$ Determined by comparing the $t_{R}$ of GC analysis and optical rotations with literature data.

\subsection{Antimicrobial Effects}

Since several aminoalcohols have been shown to exert antimicrobial activities on various bacterial and fungal strains $[74,75]$, the antimicrobial activities of the prepared aminoalcohol analogues and diol 6 were tested against two yeasts, as well as two Gram-positive and two Gram-negative bacteria (Table 2). Compounds $\mathbf{8}$ and $\mathbf{1 2}$ inhibited the studied Gram-positive bacteria with efficiencies over $20 \%$, while other derivatives showed weak activities. In the case of Bacillus subtilis, 8 showed more potential antimicrobial activity, while for Staphylococcus aureus, $\mathbf{1 2}$ proved to be the most effective agent. Furthermore, only 9 showed an inhibition activity over 30\% for Pseudomonas aeruginosa, while it had only a moderate effect against Escherichia coli. All compounds presented low-to-moderate inhibitions against $E$. coli in the range of $5-30 \%$.

Table 2. Antimicrobial activities of the synthesized compounds.

\begin{tabular}{|c|c|c|c|c|c|c|c|}
\hline \multirow[b]{3}{*}{ Analogue } & \multirow[b]{3}{*}{ Conc. $(\mu \mathrm{g} / \mathrm{mL})$} & & & \multicolumn{4}{|c|}{$\begin{array}{c}\text { Inhibitory effect }(\%) \pm \text { RSD } \\
(\%)\end{array}$} \\
\hline & & \multicolumn{2}{|c|}{ Yeast } & \multicolumn{2}{|c|}{ Gram-Negative } & \multicolumn{2}{|c|}{ Gram-Positive } \\
\hline & & C. albicans & C. krusei & E. coli & $\begin{array}{c}P . \\
\text { aeruginosa }\end{array}$ & B. subtilis & S. aureus \\
\hline \multirow{2}{*}{6} & 10 & - & $36.5 \pm 8.43$ & - & - & - & - \\
\hline & 100 & - & $58.4 \pm 14.41$ & - & - & $21.7 \pm 6.05$ & - \\
\hline \multirow{2}{*}{7} & 10 & - & - & $8.7 \pm 3.15$ & $7.5 \pm 1.54$ & - & - \\
\hline & 100 & - & - & $20.0 \pm 2.81$ & $8.7 \pm 0.49$ & - & $7.1 \pm 4.3$ \\
\hline \multirow{2}{*}{8} & 10 & - & - & - & - & $19.0 \pm 2.61$ & - \\
\hline & 100 & - & - & $17.1 \pm 4.94$ & $5.3 \pm 4.31$ & $31.9 \pm 2.74$ & - \\
\hline \multirow{2}{*}{9} & 10 & - & - & $16.7 \pm 6.68$ & $9.9 \pm 1.8$ & - & - \\
\hline & 100 & - & - & $21.0 \pm 5.05$ & $31.6 \pm 1.73$ & $9.8 \pm 11.2$ & $13.8 \pm 1.73$ \\
\hline \multirow{2}{*}{10} & 10 & - & - & $3.7 \pm 1.68$ & - & - & - \\
\hline & 100 & - & - & $4.3 \pm 10.71$ & $2.3 \pm 5.93$ & $10.5 \pm 10.12$ & - \\
\hline \multirow[b]{2}{*}{11} & 10 & - & $3.7 \pm 0.04$ & - & - & - & - \\
\hline & 100 & - & $16.0 \pm 14.5$ & - & - & - & - \\
\hline \multirow{2}{*}{12} & 10 & - & - & $15.3 \pm 4.35$ & - & - & $9.2 \pm 7.75$ \\
\hline & 100 & - & - & $26.2 \pm 4.06$ & $1.8 \pm 6.28$ & - & $20.2 \pm 8.92$ \\
\hline \multirow{2}{*}{13} & 10 & - & - & $17.1 \pm 8.19$ & - & - & - \\
\hline & 100 & - & - & $27.7 \pm 8.54$ & $7.0 \pm 4.62$ & - & $3.9 \pm 3.39$ \\
\hline \multirow{2}{*}{14} & 10 & - & - & $14.6 \pm 4.38$ & $4.1 \pm 7.10$ & - & $12.6 \pm 0.57$ \\
\hline & 100 & - & - & $25.3 \pm 2.99$ & $16.8 \pm 5.69$ & - & $14.0 \pm 3.68$ \\
\hline \multirow{2}{*}{15} & 10 & - & - & $5.1 \pm 7.92$ & - & - & - \\
\hline & 100 & - & - & $14.8 \pm 4.87$ & - & $1.5 \pm 11.4$ & - \\
\hline
\end{tabular}

According to our results, $N$-substituted 1,2-aminoalcohols 7-10 had a moderate activity against both Gram-negative and Gram-positive bacteria. Most of the ring-closing oxazolidine products (12-14) 
showed a similar moderate antibacterial activity. The removal of the nitrogen substituent of the aminoalcohols led to the loss of antibacterial activity (see amino diol 6). None of the aminoalcohol derivatives exhibited any remarkable antifungal effect, while diol 6 showed significant antifungal activity against Candida krusei (Table 2).

\section{Materials and Methods}

\subsection{Materials and General Methods}

Commercially available compounds were used as-obtained from suppliers (Molar Chemicals Ltd., Halásztelek, Hungary; Merck Ltd., Budapest, Hungary and VWR International Ltd., Debrecen, Hungary), while solvents were dried according to standard procedures. Optical rotations were measured in $\mathrm{MeOH}$ at $20^{\circ} \mathrm{C}$ with a PerkinElmer 341 polarimeter (PerkinElmer Inc., Shelton, CT, USA). Chromatographic separations and monitoring of reactions were carried out on a Merck Kieselgel 60 (Merck Ltd., Budapest, Hungary). Elemental analyses of all compounds were performed on a PerkinElmer 2400 Elemental Analyzer (PerkinElmer Inc., Waltham, MA, USA). GC measurements for the direct separation of commercially available enantiomers of isopulegol to determine the enantiomeric purity of starting material 1 and the separation of $O$-acetyl derivatives of enantiomers were performed on a Chirasil-DEX CB column $(2500 \times 0.25 \mathrm{~mm}$ I.D. $)$ on a PerkinElmer Autosystem XL GC consisting of a flame ionization detector (PerkinElmer Corporation, Norwalk, CT, USA) and a Turbochrom Workstation data system (PerkinElmer Corp., Norwalk, CT, USA). Melting points were determined on a Kofler apparatus (Nagema, Dresden, Germany) and were uncorrected. ${ }^{1} \mathrm{H}$ - and ${ }^{13} \mathrm{C}-\mathrm{NMR}$ spectra were recorded on a Brucker Avance DRX 500 spectrometer $\left[500 \mathrm{MHz}\left({ }^{1} \mathrm{H}\right)\right.$ and $125 \mathrm{MHz}\left({ }^{13} \mathrm{C}\right), \delta=0$ (TMS, Tetramethylsilane)]. Chemical shifts are expressed in ppm ( $\delta$ ) relative to TMS as the internal reference. $J$ values are given by $\mathrm{Hz}$.

(-)-Isopulegol 1 is commercially available from Merck Co with $e e=95 \%$. (+)-Neoisopulegol 2 and (-)-6-methyl-3-methylenetetrahydrofuran 3 were prepared according to literature procedures. All spectroscopic data of the synthesized compounds were similar to those described therein [55]. ${ }^{1} \mathrm{H},{ }^{13} \mathrm{C}$, HSQC, HMBC and NOESY NMR spectra of new compounds are available in Supplementary Materials.

\section{2. (2'R,3aR,6R,7aS)-6-Methylhexahydro-2H-spiro[benzofuran-3,2'-oxirane] (5)}

m-chloroperbenzoic acid ( $70 \%$ purity, $5.87 \mathrm{~g}, 23.8 \mathrm{mmol}$ ) was added at $0{ }^{\circ} \mathrm{C}$ to a solution of 3 (11.9 mmol) in $\mathrm{CH}_{2} \mathrm{Cl}_{2}(50 \mathrm{~mL})$ and $\mathrm{Na}_{2} \mathrm{HPO}_{4} \cdot 12 \mathrm{H}_{2} \mathrm{O}(6.35 \mathrm{~g}$, $35.7 \mathrm{mmol})$ in water $(130 \mathrm{~mL})$, and the mixture was stirred at room temperature. When the reaction was complete, as indicated by TLC (Thin layer chromatography) ( $2 \mathrm{~h}$ ), the mixture was separated and the aqueous phase was extracted with $\mathrm{CH}_{2} \mathrm{Cl}_{2}(100 \mathrm{~mL})$. The organic layer was washed with a $5 \% \mathrm{KOH}$ solution $(3 \times 50 \mathrm{~mL})$, then dried $\left(\mathrm{Na}_{2} \mathrm{SO}_{4}\right)$ and evaporated to provide 5 as the single product.

Yield: $23 \%$, colorless oil. $[\alpha]_{\mathrm{D}}^{20}=-26.0(\mathrm{c} 0.27, \mathrm{MeOH}) .{ }^{1} \mathrm{H} \mathrm{NMR}\left(500 \mathrm{MHz}, \mathrm{CDCl}_{3}\right): \delta=0.83-0.93$ $(1 \mathrm{H}, \mathrm{m}), 0.90(3 \mathrm{H}, \mathrm{d}, J=6.5 \mathrm{~Hz}), 1.18-1.26(1 \mathrm{H}, \mathrm{m}), 1.32-1.42(1 \mathrm{H}, \mathrm{m}), 1.55-1.75(4 \mathrm{H}, \mathrm{m}), 2.03-2.10(1 \mathrm{H}$, $\mathrm{m}), 2.83(1 \mathrm{H}, \mathrm{d}, J=4.2 \mathrm{~Hz}), 2.96(1 \mathrm{H}, \mathrm{d}, J=4.2 \mathrm{~Hz}), 3.63(1 \mathrm{H}, \mathrm{d}, J=10.6 \mathrm{~Hz}), 4.21(1 \mathrm{H}, \mathrm{d}, J=10.6 \mathrm{~Hz})$, $4.25(1 \mathrm{H}, \mathrm{d}, J=2.4 \mathrm{~Hz}) .{ }^{13} \mathrm{C} \mathrm{NMR}\left(125 \mathrm{MHz}, \mathrm{CDCl}_{3}\right): \delta=22.4,24.7,26.4,33.0,36.5,42.7,47.4,68.0,70.1$, 77.9. Anal. Calculated for $\mathrm{C}_{10} \mathrm{H}_{16} \mathrm{O}_{2}$ : $\mathrm{C}, 71.39 ; \mathrm{H}, 9.59$. Found: $\mathrm{C}, 71.43 ; \mathrm{H}, 9.52$.

\subsection{General Procedure for Ring-Opening of Epoxide with Primary Amines}

A solution of the appropriate amine $(5.88 \mathrm{mmol})$ in $\mathrm{MeCN}(10 \mathrm{~mL})$ and $\mathrm{LiClO}_{4}(0.31 \mathrm{~g}, 2.94 \mathrm{mmol})$ was added to a solution of epoxide $5(0.50 \mathrm{~g}, 2.94 \mathrm{mmol})$ in $\mathrm{MeCN}(30 \mathrm{~mL})$. The mixture was kept at reflux temperature for $6 \mathrm{~h}$. When the reaction was completed (indicated by TLC), the mixture was evaporated to dryness, and the residue was dissolved in water $(15 \mathrm{~mL})$ then extracted with $\mathrm{CH}_{2} \mathrm{Cl}_{2}$ $(3 \times 50 \mathrm{~mL})$. The combined organic phase was dried $\left(\mathrm{Na}_{2} \mathrm{SO}_{4}\right)$, filtered, and concentrated. The crude product was purified by column chromatography on silica gel with an appropriate solvent mixture 
$\left(\mathrm{CHCl}_{3}: \mathrm{MeOH}=19: 1\right)$. Further purification by recrystallization from a mixture of $n$-hexane: $\mathrm{Et}_{2} \mathrm{O}$ resulted in compounds $\mathbf{7 - 1 0 .}$

\subsection{1. (3R,3aR,6R,7aS)-6-Methyl-3-((((R)-1-phenylethyl)amino)methyl)octahydrobenzofuran-3-ol (7)}

Yield: $65 \%$, white crystals, m.p.: $77-81^{\circ} \mathrm{C} .[\alpha]_{\mathrm{D}}^{20}=+27.0(\mathrm{c} 0.25, \mathrm{MeOH}) .{ }^{1} \mathrm{H}$ NMR $(500 \mathrm{MHz}$, $\left.\mathrm{CDCl}_{3}\right): \delta=0.78-0.87(1 \mathrm{H}, \mathrm{m}), 0.86(3 \mathrm{H}, \mathrm{d}, J=6.5 \mathrm{~Hz}), 0.98-1.06(1 \mathrm{H}, \mathrm{m}), 1.12-1.18(1 \mathrm{H}, \mathrm{m}), 1.38(3 \mathrm{H}$, $\mathrm{d}, J=6.6 \mathrm{~Hz}), 1.45-1.50(1 \mathrm{H}, \mathrm{m}), 1.51-1.63(3 \mathrm{H}, \mathrm{m}), 1.66-1.72(1 \mathrm{H}, \mathrm{m}), 2.00-2.05(1 \mathrm{H}, \mathrm{m}), 2.42(1 \mathrm{H}, \mathrm{d}$, $J=12.1 \mathrm{~Hz}), 2.77(1 \mathrm{H}, \mathrm{d}, J=12.1 \mathrm{~Hz}), 3.64(1 \mathrm{H}, \mathrm{d}, J=9.5 \mathrm{~Hz}), 3.70(1 \mathrm{H}, \mathrm{d}, J=9.6 \mathrm{~Hz}), 3.79(1 \mathrm{H}, \mathrm{q}$, $J=6.5 \mathrm{~Hz}), 4.37(1 \mathrm{H}, \mathrm{q}, J=3.0 \mathrm{~Hz}), 7.25-7.35(5 \mathrm{H}, \mathrm{m}) .{ }^{13} \mathrm{C} \mathrm{NMR}\left(125 \mathrm{MHz}, \mathrm{CDCl}_{3}\right): \delta=22.4,24.4,24.5$, 26.5, 33.2, 37.0, 47.1, 49.5, 58.6, 76.3, 77.6, 82.4, 126.7, 127.4, 128.8. Anal. Calculated for $\mathrm{C}_{18} \mathrm{H}_{27} \mathrm{NO}_{2}$ : C, 74.70; H, 9.40; N, 4.84. Found: C, 74.73; H, 9.45; N, 4.80.

\subsection{2. (3R,3aR,6R,7aS)-6-Methyl-3-((((S)-1-phenylethyl)amino)methyl)octahydrobenzofuran-3-ol (8)}

Yield: $75 \%$, colorless oil. $[\alpha]_{\mathrm{D}}^{20}=-23.0(\mathrm{c} 0.255, \mathrm{MeOH}) .{ }^{1} \mathrm{H} \mathrm{NMR}\left(500 \mathrm{MHz}, \mathrm{CDCl}_{3}\right): \delta=0.75-0.85$ $(1 \mathrm{H}, \mathrm{m}), 0.85(3 \mathrm{H}, \mathrm{d}, J=6.3 \mathrm{~Hz}), 0.90-1.00(1 \mathrm{H}, \mathrm{m}), 1.10-1.16(1 \mathrm{H}, \mathrm{m}), 1.35-1.40(1 \mathrm{H}, \mathrm{m}), 1.39(3 \mathrm{H}, \mathrm{d}$, $J=6.6 \mathrm{~Hz}), 1.50-1.60(2 \mathrm{H}, \mathrm{m}), 1.63-1.67(1 \mathrm{H}, \mathrm{m}), 2.01(1 \mathrm{H}, \mathrm{d}, J=14.5 \mathrm{~Hz}), 2.46(1 \mathrm{H}, \mathrm{d}, J=12.2 \mathrm{~Hz}), 2.65$ $(1 \mathrm{H}, \mathrm{d}, J=12.2 \mathrm{~Hz}), 3.73(3 \mathrm{H}, \mathrm{dd}, J=9.5,20.2 \mathrm{~Hz}), 4.37(1 \mathrm{H}, \mathrm{s}), 7.25-7.40(5 \mathrm{H}, \mathrm{m}) .{ }^{13} \mathrm{C}$ NMR $(125 \mathrm{MHz}$, $\left.\mathrm{CDCl}_{3}\right): \delta=22.3,24.2,24.3,26.5,33.1,37.0,46.9,49.6,58.9,76.2,77.6,82.3,126.4,127.4,128.8,144.9$. Anal. Calculated for $\mathrm{C}_{18} \mathrm{H}_{27} \mathrm{NO}_{2}$ : C, 74.70; $\mathrm{H}, 9.40 ; \mathrm{N}, 4.84$. Found: $\mathrm{C}, 74.68 ; \mathrm{H}, 9.43 ; \mathrm{N}, 4.85$.

\subsection{3. (3R,3aR,6R,7aS)-3-((Benzylamino)methyl)-6-methyloctahydrobenzofuran-3-ol (9)}

Yield: $78 \%$, white crystals, m.p.: $55-56{ }^{\circ} \mathrm{C}$. $[\alpha]_{\mathrm{D}}^{20}=-7.0$ (c $\left.0.255, \mathrm{MeOH}\right) .{ }^{1} \mathrm{H}$ NMR $(500 \mathrm{MHz}$, $\left.\mathrm{CDCl}_{3}\right): \delta=0.80-0.87(1 \mathrm{H}, \mathrm{m}), 0.87(3 \mathrm{H}, \mathrm{d}, J=6.5 \mathrm{~Hz}), 1.03-1.06(1 \mathrm{H}, \mathrm{m}), 1.11-1.17(1 \mathrm{H}, \mathrm{m}), 1.45-1.49$ $(1 \mathrm{H}, \mathrm{m}), 1.55-1.62(2 \mathrm{H}, \mathrm{m}), 2.01-2.05(1 \mathrm{H}, \mathrm{m}), 2.58(1 \mathrm{H}, \mathrm{d}, J=12.1 \mathrm{~Hz}), 2.70(1 \mathrm{H}, \mathrm{brs}), 2.86(1 \mathrm{H}, \mathrm{d}$, $J=12.2 \mathrm{~Hz}), 3.70(1 \mathrm{H}, \mathrm{d}, J=9.5 \mathrm{~Hz}), 3.79(1 \mathrm{H}, \mathrm{d}, J=9.6 \mathrm{~Hz}), 3.80(1 \mathrm{H}, \mathrm{s}), 4.39(1 \mathrm{H}, \mathrm{dd}, J=3.0,6.0 \mathrm{~Hz})$, 7.25-7.35 (5H, m). ${ }^{13} \mathrm{C}$ NMR $\left(125 \mathrm{MHz}, \mathrm{CDCl}_{3}\right): \delta=22.3,24.3,26.4,33.1,36.9,47.1,51.1,54.3,76.3,77.6$, 82.4, 127.4, 128.1, 128.6, 139.7. Anal. Calculated for $\mathrm{C}_{17} \mathrm{H}_{25} \mathrm{NO}_{2}: \mathrm{C}, 74.14 ; \mathrm{H}, 9.15 ; \mathrm{N}, 5.09$. Found: $\mathrm{C}$, 74.20; H, 9.10; N, 4.05 .

\subsection{4. (3R,3aR,6R,7aS)-3-((Isopropylamino)methyl)-6-methyloctahydrobenzofuran-3-ol (10)}

Yield: $83 \%$, white crystals, m.p.: $171-173{ }^{\circ} \mathrm{C}$. $[\alpha]_{\mathrm{D}}^{20}=-7.0$ (c 0.28, MeOH). ${ }^{1} \mathrm{H}$ NMR $(500 \mathrm{MHz}$, DMSO- $\left.d_{6}\right): \delta=0.75-1.00(2 \mathrm{H}, \mathrm{m}), 0.84(3 \mathrm{H}, \mathrm{d}, J=3.5 \mathrm{~Hz}), 1.10-1.30(2 \mathrm{H}, \mathrm{m}), 1.22(6 \mathrm{H}, \mathrm{s}), 1.48(1 \mathrm{H}$, brs), $1.57(2 \mathrm{H}, \mathrm{d}, J=8.7 \mathrm{~Hz}), 1.75-1.95(2 \mathrm{H}, \mathrm{m}), 2.91(1 \mathrm{H}, \mathrm{d}, J=12.2 \mathrm{~Hz}), 3.06(1 \mathrm{H}, \mathrm{d}, J=12.3 \mathrm{~Hz})$, $3.28(1 \mathrm{H}, \mathrm{brs}), 3.60(1 \mathrm{H}, \mathrm{d}, J=8.8 \mathrm{~Hz}), 3.81(1 \mathrm{H}, \mathrm{d}, J=8.9 \mathrm{~Hz}), 4.30(1 \mathrm{H}, \mathrm{brs}) .{ }^{13} \mathrm{C}$ NMR $(125 \mathrm{MHz}$, DMSO- $\left.d_{6}\right): \delta=18.3,18.6,22.1,23.2,26.0,32.4,36.3,46.1,46.6,50.5,75.1 ., 76.4,80.1$. Anal. Calculated for $\mathrm{C}_{13} \mathrm{H}_{25} \mathrm{NO}_{2}$ : C, 68.68; H, 11.08; N, 6.16. Found: C, 68.70; H, 11.03; N, 6.18.

\subsection{General Procedure for Ring Closure of Aminoalcohols 7-10 with Formaldehyde}

Thirty-five percent aqueous formaldehyde $(20 \mathrm{~mL})$ was added to a solution of aminoalcohols 7-10 (1.8 mmol) in $\mathrm{Et}_{2} \mathrm{O}(5 \mathrm{~mL})$, and the mixture was stirred at room temperature. After $1 \mathrm{~h}$, it was made alkaline with $10 \%$ aqueous $\mathrm{KOH}(20 \mathrm{~mL})$ and extracted with $\mathrm{Et}_{2} \mathrm{O}(3 \times 50 \mathrm{~mL})$. After drying $\left(\mathrm{Na}_{2} \mathrm{SO}_{4}\right)$ and solvent evaporation, crude products $\mathbf{1 2}-\mathbf{1 5}$ were purified by column chromatography $\left(\mathrm{CHCl}_{3}: \mathrm{MeOH}=19: 1\right)$.

3.4.1. (3R,3aR,6R,7aS)-6-Methyl-3'-((R)-1-phenylethyl)hexahydro-2H-spiro[benzofuran-3,5' -oxazolidine] (12)

Yield: $50 \%$, colorless oil. $[\alpha]_{\mathrm{D}}^{20}=+27.0(\mathrm{c}, 0.275 \mathrm{MeOH}) .{ }^{1} \mathrm{H}$ NMR $\left(500 \mathrm{MHz}, \mathrm{CDCl}_{3}\right): \delta=0.84-0.95$ $(2 \mathrm{H}, \mathrm{m}), 0.87(3 \mathrm{H}, \mathrm{d}, J=6.3 \mathrm{~Hz}), 1.10-1.17(1 \mathrm{H}, \mathrm{m}), 1.34(3 \mathrm{H}, \mathrm{d}, J=6.4 \mathrm{~Hz}), 1.50-1.65(3 \mathrm{H}, \mathrm{m}), 1.78-1.83$ $(1 \mathrm{H}, \mathrm{m}), 2.02(1 \mathrm{H}, \mathrm{d}, J=14.4 \mathrm{~Hz}), 2.57(1 \mathrm{H}, \mathrm{d}, J=10.5 \mathrm{~Hz}), 2.94(1 \mathrm{H}, \mathrm{d}, J=10.6 \mathrm{~Hz}), 3.35-3.40(1 \mathrm{H}$, 
$\mathrm{m}), 3.88(2 \mathrm{H}, \mathrm{dd}, J=9.7,19.1 \mathrm{~Hz}), 4.24-4.30(3 \mathrm{H}, \mathrm{m}), 7.22-7.33(5 \mathrm{H}, \mathrm{m}) .{ }^{13} \mathrm{C} \mathrm{NMR}\left(125 \mathrm{MHz}, \mathrm{CDCl}_{3}\right)$ : $\delta=22.3,23.4,24.6,26.3,33.2,36.9,45.7,53.3,62.5,76.4,78.3,84.6,91.5,127.2,127.4,128.6,144.8$. Anal. Calculated for $\mathrm{C}_{19} \mathrm{H}_{27} \mathrm{NO}_{2}$ : C, 75.71; $\mathrm{H}, 9.03 ; \mathrm{N}, 4.65$. Found: $\mathrm{C}, 75.73 ; \mathrm{H}, 9.00 ; \mathrm{N}, 4.68$.

3.4.2. (3R,3aR,6R,7aS)-6-Methyl-3'-((S)-1-phenylethyl)hexahydro-2H-spiro[benzofuran-3,5' -oxazolidine] (13)

Yield: $95 \%$, colorless oil. $[\alpha]_{\mathrm{D}}^{20}=-27.0(\mathrm{c}, 0.25 \mathrm{MeOH}) .{ }^{1} \mathrm{H} \mathrm{NMR}\left(500 \mathrm{MHz}, \mathrm{CDCl}_{3}\right): \delta=0.82-0.96$ $(3 \mathrm{H}, \mathrm{m}), 0.87(3 \mathrm{H}, \mathrm{d}, J=6.3 \mathrm{~Hz}), 1.12-1.20(1 \mathrm{H}, \mathrm{m}), 1.25(1 \mathrm{H}, \mathrm{s}), 1.36(3 \mathrm{H}, \mathrm{d}, J=6.2 \mathrm{~Hz}), 1.54-1.65$ $(3 \mathrm{H}, \mathrm{m}), 1.80-1.85(2 \mathrm{H}, \mathrm{m}), 2.02(1 \mathrm{H}, \mathrm{d}, J=14.4 \mathrm{~Hz}), 2.53(1 \mathrm{H}, \mathrm{d}, J=10.6 \mathrm{~Hz}), 2.94(1 \mathrm{H}, \mathrm{d}, J=10.7 \mathrm{~Hz})$, 3.35-3.45 (1H, m), $3.87(2 \mathrm{H}, \mathrm{t}, J=10.7 \mathrm{~Hz}), 4.26(2 \mathrm{H}, \mathrm{s}), 4.36(1 \mathrm{H}, \mathrm{s}), 7.20-7.40(5 \mathrm{H}, \mathrm{m}) .{ }^{13} \mathrm{C} \mathrm{NMR}$ $\left(125 \mathrm{MHz}_{\mathrm{CDCl}}\right): \delta=22.3,23.5,24.5,26.3,33.2,36.8,45.7,53.4,62.5,76.2,78.2,84.8,127.2,127.4,128.7$. Anal. Calculated for $\mathrm{C}_{19} \mathrm{H}_{27} \mathrm{NO}_{2}$ : C, 75.71; H, 9.03; N, 4.65. Found: C, 75.70; H, 9.07; N, 4.63.

\subsection{3. (3R,3aR,6R,7aS)-3'-Benzyl-6-methylhexahydro-2H-spiro[benzofuran-3,5' -oxazolidine] (14)}

Yield: $90 \%$, white crystals, m.p.: $76-77^{\circ} \mathrm{C} .[\alpha]_{\mathrm{D}}^{20}=-9.0(\mathrm{c}, 0.25 \mathrm{MeOH}) .{ }^{1} \mathrm{H} \mathrm{NMR}\left(500 \mathrm{MHz}, \mathrm{CDCl}_{3}\right)$ : $\delta=0.84-1.00(2 \mathrm{H}, \mathrm{m}), 0.88(3 \mathrm{H}, \mathrm{d}, J=6.5 \mathrm{~Hz}), 1.14-1.21(1 \mathrm{H}, \mathrm{m}), 1.55-1.65(3 \mathrm{H}, \mathrm{m}), 1.80-1.84(1 \mathrm{H}, \mathrm{m})$, $2.04(1 \mathrm{H}, \mathrm{d}, J=13.8 \mathrm{~Hz}), 2.70(1 \mathrm{H}, \mathrm{d}, J=11.8 \mathrm{~Hz}), 3.09(1 \mathrm{H}, \mathrm{d}, J=11.8 \mathrm{~Hz}), 3.68(2 \mathrm{H}, \mathrm{dd}, J=13.0$, $18.4 \mathrm{~Hz}), 3.91(2 \mathrm{H}, \mathrm{dd}, J=9.8,11.2 \mathrm{~Hz}), 4.31(1 \mathrm{H}, \mathrm{d}, J=2.7 \mathrm{~Hz}), 4.35(2 \mathrm{H}, \mathrm{s}), 7.25-7.35(5 \mathrm{H}, \mathrm{m}) .{ }^{13} \mathrm{C} \mathrm{NMR}$ $\left(125 \mathrm{MHz}_{\mathrm{CDCl}}\right): \delta=22.3,24.6,26.3,33.2,36.8,46.1,54.5,58.7,76.7,78.6,86.0,90.5,127.5,128.6,128.8$, 138.6. Anal. Calculated for $\mathrm{C}_{18} \mathrm{H}_{25} \mathrm{NO}_{2}$ : C, 75.22; $\mathrm{H}, 8.77 ; \mathrm{N}, 4.87$. Found: C, 75.25; H, 9.73; N, 4.90.

3.4.4. (3R,3aR,6R,7aS)-3'-Isopropyl-6-methylhexahydro-2H-spiro[benzofuran-3,5'-oxazolidine] (15)

Yield: $95 \%$, colorless oil. $[\alpha]_{\mathrm{D}}^{20}=-13.0(\mathrm{c} 0.25, \mathrm{MeOH}) .{ }^{1} \mathrm{HNMR}\left(500 \mathrm{MHz}, \mathrm{DMSO}-d_{6}\right): \delta=0.79-0.86$ $(1 \mathrm{H}, \mathrm{m}), 0.83(3 \mathrm{H}, \mathrm{d}, J=6.6 \mathrm{~Hz}), 0.89-1.01(2 \mathrm{H}, \mathrm{m}), 0.99(6 \mathrm{H}, \mathrm{d}, J=6.2 \mathrm{~Hz}), 1.07-1.13(1 \mathrm{H}, \mathrm{m}), 1.45-1.55$ $(1 \mathrm{H}, \mathrm{m}), 1.55-1.60(2 \mathrm{H}, \mathrm{m}), 1.68-1.73(1 \mathrm{H}, \mathrm{m}), 1.86(1 \mathrm{H}, \mathrm{d}, J=14.2 \mathrm{~Hz}), 2.35-2.40(1 \mathrm{H}, \mathrm{m}), 2.60(1 \mathrm{H}$, $\mathrm{d}, J=10.1 \mathrm{~Hz}), 2.87(1 \mathrm{H}, \mathrm{d}, J=10.2 \mathrm{~Hz}), 3.64(1 \mathrm{H}, \mathrm{d}, J=9.6 \mathrm{~Hz}), 3.84(1 \mathrm{H}, \mathrm{d}, J=9.6 \mathrm{~Hz}), 4.09(1 \mathrm{H}, \mathrm{d}$, $J=2.6 \mathrm{~Hz}), 4.18(1 \mathrm{H}, \mathrm{d}, J=3.2 \mathrm{~Hz}), 4.20(1 \mathrm{H}, \mathrm{d}, J=3.2 \mathrm{~Hz}) .{ }^{13} \mathrm{C} \mathrm{NMR}\left(125 \mathrm{MHz}, \mathrm{DMSO}-d_{6}\right): \delta=21.7$, 21.8, 22.2, 23.7, 25.8, 32.5, 36.4, 44.9, 51.8, 51.9, 75.4, 77.1, 83.6, 91.1. Anal. Calculated for $\mathrm{C}_{14} \mathrm{H}_{25} \mathrm{NO}_{2}: \mathrm{C}_{\text {, }}$ 70.25; H, 10.53; N, 5.85. Found: C, 70.28; H, 10.50; N, 5.83 .

\section{5. (3R,3aR,6R,7aS)-3-(Aminomethyl)-6-methyloctahydrobenzofuran-3-ol (11)}

Aminoalcohols 7-9 (14.0 $\mathrm{mmol})$ in $\mathrm{MeOH}(100 \mathrm{~mL})$ were added to a suspension of palladium-on-carbon $(5 \% \mathrm{Pd}, 0.22 \mathrm{~g})$ in $\mathrm{MeOH}(50 \mathrm{~mL})$, and the mixture was stirred under an $\mathrm{H}_{2}$ atmosphere (1 atm) at room temperature. After the completion of the reaction (as monitored by TLC, $24 \mathrm{~h}$ ), the mixture was filtered through a Celite pad, and the solution was evaporated to dryness. The crude product was recrystallized in $\mathrm{Et}_{2} \mathrm{O}$, resulting in primary aminoalcohol 11.

Yield: $73 \%$ (with 7); 75\% (with 8); 70\% (with 9), white crystals, m.p.: $217-221^{\circ} \mathrm{C} .[\alpha]_{\mathrm{D}}^{20}=+7.0$ (c $0.25, \mathrm{MeOH}) .{ }^{1} \mathrm{H}$ NMR $\left(500 \mathrm{MHz}, \mathrm{DMSO}-d_{6}\right): \delta=0.74-0.89(2 \mathrm{H}, \mathrm{m}), 0.83(3 \mathrm{H}, \mathrm{d}, J=5.7 \mathrm{~Hz}), 1.46(1 \mathrm{H}$, brs), 1.53-1.65 (2H, m), 1.75-1.83 (1H, m), $1.87(1 \mathrm{H}, \mathrm{d}, J=13.8 \mathrm{~Hz}), 2.83(1 \mathrm{H}, \mathrm{d}, J=12.9 \mathrm{~Hz}), 2.95(1 \mathrm{H}, \mathrm{d}$, $J=12.9 \mathrm{~Hz}), 3.56(1 \mathrm{H}, \mathrm{d}, J=9.2 \mathrm{~Hz}), 3.80(1 \mathrm{H}, \mathrm{d}, J=9.2 \mathrm{~Hz}), 4.28(1 \mathrm{H}, \mathrm{s}), 5.45(1 \mathrm{H}, \mathrm{s}), 8.04(3 \mathrm{H}, \mathrm{s}) .{ }^{13} \mathrm{C}$ NMR (125 MHz, DMSO-d $\left.d_{6}\right): \delta=22.2,23.2,25.9,32.4,36.4,41.8,45.7,74.9,76.5,80.3$. Anal. Calculated for $\mathrm{C}_{10} \mathrm{H}_{19} \mathrm{NO}_{2}$ : C, 64.83; $\mathrm{H}, 10.34 ; \mathrm{N}, 7.56$. Found: $\mathrm{C}, 64.85 ; \mathrm{H}, 10.32 ; \mathrm{N}, 7.60$.

\section{6. (3R,3aR,6R,7aS)-3-(Hydroxymethyl)-6-methyloctahydrobenzofuran-3-ol (6)}

An aqueous solution of $\mathrm{NMO}\left(12 \mathrm{~mL}, 50 \%\right.$ aqueous solution) and a solution of $\mathrm{OsO}_{4}$ in $t$-BuOH (6 mL, $2 \% \mathrm{t}$-BuOH solution) were added in one portion to a solution of compound $3(2.13 \mathrm{~g}, 14 \mathrm{mmol}) \mathrm{in}$ acetone $(60 \mathrm{~mL})$. The reaction mixture was stirred at room temperature for $24 \mathrm{~h}$, then quenched by the addition of a saturated aqueous solution of $\mathrm{Na}_{2} \mathrm{SO}_{3}(100 \mathrm{~mL})$, and extracted with EtOAc (Ethyl acetate, $3 \times 80 \mathrm{~mL})$. The organic layer was dried $\left(\mathrm{Na}_{2} \mathrm{SO}_{4}\right)$ and evaporated. The crude product was purified 
by chromatography on silica gel by using $n$-hexane:EtOAc $=1: 4$. The product after purification was recrystallized in $\mathrm{Et}_{2} \mathrm{O}$ resulting in compound $\mathbf{6}$ as white crystals.

Yield: $50 \%$, white crystals, m.p.: $67-68^{\circ} \mathrm{C} .[\alpha]_{\mathrm{D}}^{20}=+3.0$ (c $\left.0.27, \mathrm{MeOH}\right) .{ }^{1} \mathrm{H}$ NMR $(500 \mathrm{MHz}$, DMSO- $\left.d_{6}\right): \delta=0.75-0.80(1 \mathrm{H}, \mathrm{m}), 0.82(3 \mathrm{H}, \mathrm{d}, J=6.5 \mathrm{~Hz}), 0.94-1.03(1 \mathrm{H}, \mathrm{m}), 1.04-1.11(1 \mathrm{H}, \mathrm{m}), 1.40-1.50$ $(1 \mathrm{H}, \mathrm{m}), 1.50-1.57(2 \mathrm{H}, \mathrm{m}), 1.62-1.67(1 \mathrm{H}, \mathrm{m}), 1.85(1 \mathrm{H}, \mathrm{d}, J=14.3 \mathrm{~Hz}), 3.34-3.38(1 \mathrm{H}, \mathrm{m}), 3.42(1 \mathrm{H}, \mathrm{d}$, $J=9.2 \mathrm{~Hz}), 3.47(1 \mathrm{H}, \mathrm{dd}, J=5.5,11.1 \mathrm{~Hz}), 3.68(1 \mathrm{H}, \mathrm{d}, J=9.2 \mathrm{~Hz}), 4.23(1 \mathrm{H}, \mathrm{d}, J=2.2 \mathrm{~Hz}), 4.49(1 \mathrm{H}, \mathrm{s})$, $4.52(1 \mathrm{H}, \mathrm{t}, J=5.4 \mathrm{~Hz}) .{ }^{13} \mathrm{C}$ NMR $\left(125 \mathrm{MHz}\right.$, DMSO- $\left.d_{6}\right): \delta=22.3,23.4,26.2,32.8,36.7,46.2,63.2,74.8$, 76.5, 83.5. Anal. Calculated for $\mathrm{C}_{10} \mathrm{H}_{18} \mathrm{O}_{3}$ : C, 64.49; H, 9.74. Found: $\mathrm{C}, 64.55 ; \mathrm{H}, 9.69$.

\subsection{General Procedure for the Reaction of Benzaldehyde with Diethylzinc in the Presence of Chiral Catalysts}

To the respective catalyst $(0.1 \mathrm{mmol}), 1 \mathrm{M} \mathrm{Et}_{2} \mathrm{Zn}$ in an $n$-hexane solution $(3 \mathrm{~mL}, 3 \mathrm{mmol})$ was added under argon atmosphere at room temperature. The solution was stirred for $25 \mathrm{~min}$ at room temperature, and then benzaldehyde $(1 \mathrm{mmol})$ was added. After stirring at room temperature for a further $20 \mathrm{~h}$, the reaction was quenched with a saturated $\mathrm{NH}_{4} \mathrm{Cl}$ solution $(15 \mathrm{~mL})$, and the mixture was extracted with EtOAc $(2 \times 20 \mathrm{~mL})$. The combined organic phase was washed with $\mathrm{H}_{2} \mathrm{O}(10 \mathrm{~mL})$, dried $\left(\mathrm{Na}_{2} \mathrm{SO}_{4}\right)$ and evaporated under vacuum. The obtained crude secondary alcohols were purified by flash column chromatography ( $n$-hexane:EtOAc $=4: 1$ ). The $e e$ and absolute configuration of the resulting material were determined by chiral GC on a Chirasil-DEX CB column after $O$-acetylation in $\mathrm{Ac}_{2} \mathrm{O} / \mathrm{DMPA} / \mathrm{pyridine}$.

\subsection{Antimicrobial Analyses}

For the antimicrobial analyses, the pure synthesized compounds were dissolved in $\mathrm{MeOH}$ and diluted with $\mathrm{H}_{2} \mathrm{O}$ to reach concentration levels up to 400 and $40 \mu \mathrm{g} / \mathrm{mL}$ with a final MeOH content of $10 \%$. Then, these test solutions were investigated in a microdilution assay with two Gram-positive bacteria (Bacillus subtilis SZMC 0209 and Staphylococcus aureus SZMC 14611), two Gram-negative bacteria (Escherichia coli SZMC 6271 and Pseudomonas aeruginosa SZMC 23290), and two yeast strains (Candida albicans SZMC 1533 and C. krusei SZMC 1352) according to the M07-A10 CLSI guideline [76] and our previous work $[57,77]$. For the assay, the suspensions of the microbes were prepared from overnight cultures that were cultivated in a ferment broth (bacteria: $10 \mathrm{~g} / \mathrm{L}$ peptone, $5 \mathrm{~g} / \mathrm{L} \mathrm{NaCl}$, $5 \mathrm{~g} / \mathrm{L}$ yeast extract; yeast: $20 \mathrm{~g} / \mathrm{L}$ peptone, $10 \mathrm{~g} / \mathrm{L}$ yeast extract, and $20 \mathrm{~g} / \mathrm{L}$ glucose) at $37^{\circ} \mathrm{C}$, and their concentrations were set to $2 \times 10^{5}$ cells $/ \mathrm{mL}$ with sterile media. Then, 96 -well plates were prepared by dispensing $100 \mu \mathrm{L}$ of suspension containing the bacterial or yeast cells, $50 \mu \mathrm{L}$ of sterile broth, and $50 \mu \mathrm{L}$ of the test solutions into each well, which were then incubated for $24 \mathrm{~h}$ at $37^{\circ} \mathrm{C}$. The mixture of $150 \mu \mathrm{L}$ of broth and $50 \mu \mathrm{L}$ of $10 \% \mathrm{MeOH}$ was used as the blank sample for background correction, while $100 \mu \mathrm{L}$ of the microbial suspension supplemented with $50 \mu \mathrm{L}$ of the sterile broth and $50 \mu \mathrm{L}$ of $10 \% \mathrm{MeOH}$ was applied as the negative control. The positive control contained ampicillin (Sigma) or nystatin (Sigma) for bacteria or fungi, respectively, at two concentration levels $(100 \mu \mathrm{g} / \mathrm{mL}$ and $10 \mu \mathrm{g} / \mathrm{mL}$ ). The inhibitory effects of each derivative were spectrophotometrically determined at $620 \mathrm{~nm}$ after incubation, and the inhibition rate was calculated as the percentage of the positive control after blank correction.

\section{Conclusions}

A new library of neoisopulegol-based chiral 1,2-aminoalcohols and a diol were developed from (+)-neoisopulegol, as derived from commercially available (-)-isopulegol. The obtained aminoalcohols and diol may serve as useful building blocks for the synthesis of new heterocyclic ring systems and biologically active compounds.

The in vitro antimicrobial studies have clearly shown that the resulting $N$-substituted aminoalcohols possess moderate antibacterial action on different bacterial strains, while the diol has a remarkable antifungal effect. 
Aminoalcohol derivatives were also applied as chiral catalysts in the enantioselective addition of diethylzinc to benzaldehyde with moderate but opposite enantioselectivity.

Supplementary Materials: The following are available online, Figures S3-S31: 1H, 13C, HSQC, HMBC and NOESY NMR spectra of new compounds.

Author Contributions: The listed authors contributed to this work as described in the following. Z.S., T.M.L. and A.S. designed, planned the research and interpreted the results. F.Z.B. and B.V. carried out the synthetic work. F.Z.B. and T.M.L. discussed the results and contributed to the writing of the paper. All authors discussed the results, and they also prepared and commented on the manuscript. All authors have read and agreed to the published version of the manuscript.

Funding: This research was funded by EU-funded Hungarian grant GINOP, grant number GINOP-2.3.2-15-2016-00014. The APC was funded by University of Szeged Open Access Fund' (FundRef, Grant number 4479).

Acknowledgments: Z.S. is grateful for financial support from University of Szeged Open Access Fund' (FundRef, Grant number 4479) and the EU-funded Hungarian grant GINOP-2.3.2-15-2016-00014.

Conflicts of Interest: The authors declare no conflict of interest.

\section{References}

1. Keay, B.A.; Hopkins, J.M.; Dibble, P.W. Furans and their benzo derivatives: Applications. In Comprehensive Heterocyclic Chemistry III, 1st ed.; Katritzky, A.R., Ramsden, C.A., Scriven, E.F.V., Taylor, R.J.K., Eds.; Elsevier: Amsterdam, The Netherlands, 2008; Volume 5, pp. 571-623.

2. Keay, B.A.; Dibble, P.W. Furans and their benzo derivatives: Applications. In Comprehensive Heterocyclic Chemistry II, 2nd ed.; Katritzky, A.R., Rees, C.V., Scriven, E.F.V., Eds.; Elsevier: Amsterdam, The Netherlands, 1996; Volume 2, pp. 395-436.

3. Leibeling, M.; Koester, D.C.; Pawliczek, M.; Schild, S.C.; Werz, D.B. Domino access to highly substituted chromans and isochromans from carbohydrates. Nat. Chem. Biol. 2010, 6, 199-201. [CrossRef] [PubMed]

4. Zhang, Y.; Negishi, E. Metal-promoted cyclization. 25. Palladium-catalyzed cascade carbometalation of alkynes and alkenes as an efficient route to cyclic and polycyclic structures. J. Am. Chem. Soc. 1989, 111, 3454-3456. [CrossRef]

5. Huang, Q.; Larock, R.C. Synthesis of substituted Naphthalenes and Carbazoles by the Palladium-catalyzed annulation of internal alkynes. J. Org. Chem. 2003, 68, 7342-7349. [CrossRef] [PubMed]

6. Manarin, F.; Roehrs, J.A.; Gay, R.M.; Brandão, R.; Menezes, P.H.; Nogueira, C.W.; Zeni, G. Electrophilic cyclization of 2-Chalcogenealkynylanisoles: Versatile access to 2-Chalcogen-benzo[b]furans. J. Org. Chem. 2009, 74, 2153-2162. [CrossRef] [PubMed]

7. Isono, N.; Lautens, M. Rhodium(I)-catalyzed cyclization reaction of o-alkynyl Phenols and Anilines. Domino approach to 2,3-disubstituted Benzofurans and Indoles. Org. Lett. 2009, 11, 1329-1331. [CrossRef]

8. Zhao, D.; Wu, N.; Zhang, S.; Xi, P.; Su, X.; Lan, J.; You, J. Synthesis of phenol, aromatic ether, and Benzofuran derivatives by Copper-catalyzed hydroxylation of aryl halides. Angew. Chem. Int. Ed. 2009, 48, 8729-8732. [CrossRef]

9. Shen, Z.; Dong, V.M. Benzofurans prepared by C-H Bond functionalization with acylsilanes. Angew. Chem. Int. Ed. 2009, 48, 784-786. [CrossRef]

10. Huang, X.-C.; Liu, Y.-L.; Liang, Y.; Pi, S.-F.; Wang, F.; Li, J.-H. Cycloaddition of arynes with Iodonium Ylides: A mild and general route for the synthesis of Benzofuran derivatives. Org. Lett. 2008, 10, 1525-1528. [CrossRef]

11. Kokubo, K.; Harada, K.; Mochizuki, E.; Oshima, T. A new approach to benzofuran synthesis: Lewis acid mediated cycloaddition of benzoquinones with stilbene oxides. Tetrahedron Lett. 2010, 51, 955-958. [CrossRef]

12. Baguley, P.A.; Jackson, L.V.; Walton, J.C. Preparation of 1-phenylcyclohexa-2,5-diene-1-carboxylates and their use in free-radical mediated syntheses. J. Chem. Soc. Perkin 1 2002, 304-309. [CrossRef] 
13. McCarroll, A.J.; Walton, J.C. Enhanced radical delivery from aldoxime esters for EPR and ring closure applications. Chem. Commun. 2000, 351-352. [CrossRef]

14. Harvey, W.E.; Tarbell, D.S. The reaction of the magnesium salt of N-cyclohexylcyclohexylimine with epoxides. J. Org. Chem. 1967, 32, 1679-1681. [CrossRef]

15. Coaviche-Yoval, A.; Andrade-Jorge, E.; Pérez-González, C.; Luna, H.; Tovar-Miranda, R.; Trujillo-Ferrara, J.G. Quantum reality in the selective reduction of a Benzofuran system. Molecules 2019, 24, 2061. [CrossRef] [PubMed]

16. Yakura, T.; Yamada, S.; Shima, M.; Iwamoto, M.; Ikeda, M. Synthesis of Octahydrobenzo[b]furans using Tandem conjugate addition reactions initiated by oxygen nucleophile. Chem. Pharm. Bull. (Tokyo) 1998, 46, 744-748. [CrossRef]

17. Ferraz, H.M.C.; Longo, L.S. Bicyclic $\beta$-hydroxytetrahydrofurans as precursors of medium ring keto-lactones. J. Org. Chem. 2007, 72, 2945-2950. [CrossRef]

18. Herrinton, P.H.; Hopkins, M.H.; Mishra, P.; Brown, M.J.; Overman, L.E. Ring-enlarging furan annulations. J. Org. Chem. 1987, 52, 3711-3712. [CrossRef]

19. Groves, J.T. A stereochemical probe of the fate of carbon radicals oxidized by metals. Tetrahedron Lett. 1975, 16, 3113-3116. [CrossRef]

20. Sohail, M.; Wang, Y.-F.; Wu, S.; Zeng, W.; Chen, F.-X. Asymmetric synthesis of octahydrobenzofuran core structure with three contiguous stereogenic centers and development of the absolute configurations. Synth. Commun. 2014, 44, 115-120. [CrossRef]

21. Arimitsu, K.; Nomura, S.; Iwasaki, H.; Ozeki, M.; Yamashita, M. First total synthesis of ( \pm )-adunctin B. Tetrahedron Lett. 2011, 52, 7046-7048. [CrossRef]

22. Trost, B.M.; Shen, H.C.; Surivet, J.-P. An enantioselective biomimetic total synthesis of (-)-Siccanin. Angew. Chem. Int. Ed. 2003, 42, 3943-3947. [CrossRef]

23. Bhagavathula, D.; Boddeti, G.; Reddy, V. A brief review on synthesis of $\beta$-amino alcohols by ring opening of epoxides. Res. Rev. J. Chem. 2017, 6, 27-46.

24. Brik, A.; Wong, C.-H. HIV-1 protease: Mechanism and drug discovery. Org. Biomol. Chem. 2003, 1, 5-14. [CrossRef] [PubMed]

25. Ghosh, A.K.; Bilcer, G.; Schiltz, G. Syntheses of FDA ppproved HIV protease inhibitors. Synthesis 2001, 2001, 2203-2229. [CrossRef] [PubMed]

26. Andrews, K.T.; Fairlie, D.P.; Madala, P.K.; Ray, J.; Wyatt, D.M.; Hilton, P.M.; Melville, L.A.; Beattie, L.; Gardiner, D.L.; Reid, R.C.; et al. Potencies of human immunodeficiency virus protease inhibitors in vitro against Plasmodium falciparum and in vivo against murine malaria. Antimicrob. Agents Chemother. 2006, 50, 639-648. [CrossRef]

27. Nöteberg, D.; Hamelink, E.; Hultén, J.; Wahlgren, M.; Vrang, L.; Samuelsson, B.; Hallberg, A. Design and synthesis of plasmepsin I and plasmepsin II inhibitors with activity in Plasmodium falciparum-infected cultured human erythrocytes. J. Med. Chem. 2003, 46, 734-746. [CrossRef]

28. Parikh, S.; Gut, J.; Istvan, E.; Goldberg, D.E.; Havlir, D.V.; Rosenthal, P.J. Antimalarial cctivity of human immunodeficiency virus type 1 protease inhibitors. Antimicrob. Agents Chemother. 2005, 49, 2983-2985. [CrossRef]

29. Savoia, D.; Allice, T.; Tovo, P.-A. Antileishmanial activity of HIV protease inhibitors. Int. J. Antimicrob. Agents 2005, 26, 92-94. [CrossRef]

30. Conolly, M.E.; Kersting, F.; Dollery, C.T. The clinical pharmacology of beta-adrenoceptor-blocking drugs. Prog. Cardiovasc. Dis. 1976, 19, 203-234. [CrossRef]

31. Shanks, R.G.; Wood, T.M.; Dornhorst, A.C.; Clark, M.L. Some pharmacological properties of a new adrenergic $\beta$-receptor antagonist. Nature 1966, 212, 88-90. [CrossRef]

32. Zimmerman, T.J.; Boger, W.P. The beta-adrenergic blocking agents and the treatment of glaucoma. Surv. Ophthalmol. 1979, 23, 347-362. [CrossRef]

33. Ager, D.J.; Prakash, I.; Schaad, D.R. 1,2-Amino alcohols and their heterocyclic derivatives as chiral auxiliaries in asymmetric synthesis. Chem. Rev. 1996, 96, 835-876. [CrossRef] [PubMed]

34. Griesbeck, A.G.; Lex, J.; Saygin, K.M.; Steinwascher, J. Azidohydroperoxidation of pinenes: Stereoselectivity pattern and the first X-ray structure of a 2-azidohydroperoxide. Chem. Commun. 2000, 2205-2206. [CrossRef] 
35. Frensch, G.; Labes, R.; Wosch, C.L.; Munaretto, L.D.S.; Salomé, K.S.; Guerrero, P.G.; Marques, F.A. New chiral ligands derived from $(+)$ and $(-)-\alpha$-pinene for the enantioselective addition of diethylzinc to aldehydes. Tetrahedron Lett. 2016, 57, 420-422. [CrossRef]

36. Hobuß, D.; Baro, A.; Laschat, S.; Frey, W. Catalytic enantioselective borane reduction of arylketones with pinene-derived amino alcohols. Tetrahedron 2008, 64, 1635-1640. [CrossRef]

37. Masui, M.; Shioiri, T. A practical method for preparation of optically pure oxazaborolidines from $\alpha$-Pinene. Tetrahedron 1995, 51, 8363-8370. [CrossRef]

38. Boobalan, R.; Chang, Y.-M.; Chen, C.; Lee, G.-H. Copper complex of Pinene based Schiff base [CuSBADBH] ${ }_{2}$ : Synthesis and its application in catalytic asymmetric nitroaldol (Henry) reaction. ChemistrySelect 2016, 1, 2028-2034. [CrossRef]

39. Łączkowski, K.Z.; Kmieciak, A.; Kozakiewicz, A. Stereoselective synthesis of new monoterpene $\beta$-amino alcohols. Tetrahedron Asymmetry 2009, 20, 1487-1492. [CrossRef]

40. Banina, O.A.; Sudarikov, D.V.; Nigmatov, A.G.; Frolova, L.L.; Slepukhin, P.A.; Zlotin, S.G.; Kutchin, A.V. Carane amino alcohols as organocatalysts in asymmetric aldol reaction of isatin with acetone. Russ. Chem. Bull. 2017, 66, 293-296. [CrossRef]

41. Rafiński, Z.; Krzemiński, M.P. Synthesis of (-)-Verbenone-derived triazolium salts and their application in enantioselective intramolecular Stetter reaction. Catalysts 2019, 9, 117. [CrossRef]

42. Frolova, L.L.; Sudarikov, D.V.; Alekseev, I.N.; Banina, O.A.; Slepukhin, P.A.; Kutchin, A.V. Synthesis of new enantiomerically pure $\beta$-amino alcohols of the pinane series. Russ. J. Org. Chem. 2017, 53, 335-343. [CrossRef]

43. Dimitrov, V.; Dobrikov, G.; Genov, M. Chiral $\beta$ - and $\gamma$-aminoalcohols derived from (+)-camphor and (-)-fenchone as catalysts for the enantioselective addition of diethylzinc to benzaldehyde. Tetrahedron Asymmetry 2001, 12, 1323-1329. [CrossRef]

44. Rafiński, Z. Enantioselective benzoin condensation catalyzed by spirocyclic terpene-based $N$-heterocyclic carbenes. Tetrahedron 2016, 72, 1860-1867. [CrossRef]

45. Rafiński, Z.; Kozakiewicz, A.; Rafińska, K. Highly efficient synthesis of spirocyclic (1R)-camphor-derived triazolium salts: Application in the catalytic asymmetric benzoin condensation. Tetrahedron 2014, 70, 5739-5745. [CrossRef]

46. Wilkinson, H.S.; Grover, P.T.; Vandenbossche, C.P.; Bakale, R.P.; Bhongle, N.N.; Wald, S.A.; Senanayake, C.H. A new lithium alkoxide accelerated diastereoselective cyanation of ketones. Org. Lett. 2001, 3, 553-556. [CrossRef] [PubMed]

47. Panev, S.; Linden, A.; Dimitrov, V. Chiral aminoalcohols with a menthane skeleton as catalysts for the enantioselective addition of diethylzinc to benzaldehyde. Tetrahedron Asymmetry 2001, 12, 1313-1321. [CrossRef]

48. Friedrich, D.; Bohlmann, F. Total synthesis of various elemanolides. Tetrahedron 1988, 44, $1369-1392$. [CrossRef]

49. Rigamonti, M.G.; Gatti, F.G. Stereoselective synthesis of hernandulcin, peroxylippidulcine A, lippidulcines A, B and C and taste evaluation. Beilstein J. Org. Chem. 2015, 11, 2117-2124. [CrossRef]

50. Moreira, J.A.; Corrêa, A.G. Enantioselective synthesis of three stereoisomers of 5,9-dimethylpentadecane, sex pheromone component of Leucoptera coffeella, from (-)-isopulegol. Tetrahedron Asymmetry 2003, 14, 3787-3795. [CrossRef]

51. Nazimova, E.; Pavlova, A.; Mikhalchenko, O.; Il'ina, I.; Korchagina, D.; Tolstikova, T.; Volcho, K.; Salakhutdinov, N. Discovery of highly potent analgesic activity of isopulegol-derived

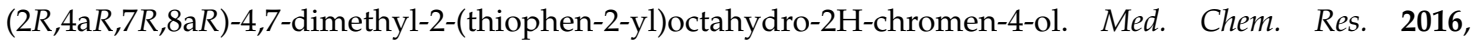
25, 1369-1383. [CrossRef]

52. Engel, W. In vivo studies on the metabolism of the monoterpene Pulegone in humans using the metabolism of ingestion-correlated amounts (MICA) approach: Explanation for the toxicity differences between $(S)-(-)$ and (R)-(+)-Pulegone. J. Agric. Food Chem. 2003, 51, 6589-6597. [CrossRef]

53. Bulliard, M.; Balme, G.; Gore, J. Fragmentation of isopulegol by a radical process. Tetrahedron Lett. 1989, 30, 2213-2216. [CrossRef]

54. Hegde, S.G.; Beckwith, D.; Doti, R.; Wolinsky, J. Synthesis with hypochlorous acid. Conversion to pulegone and isopulegol to menthofuran. Preparation of 3,6-dimethyl-2,6-cycloheptadien-1-one from phorone. J. Org. Chem. 1985, 50, 894-896. [CrossRef] 
55. Brocksom, T.J.; dos Santos, R.B.; Varanda, N.A.; Brocksom, U. An efficient synthesis of monoterpene $\alpha$-methylene- $\gamma$-butyrolactones. Synth. Commun. 1988, 18, 1403-1410. [CrossRef]

56. Schlosser, M.; Kotthaus, M. Isopulegol as a model compound: Metalation and substitution of an allylic position in the presence of an unprotected hydroxy function. Eur. J. Org. Chem. 1999, 1999, 459-462. [CrossRef]

57. Le, T.M.; Szilasi, T.; Volford, B.; Szekeres, A.; Fülöp, F.; Szakonyi, Z. Stereoselective synthesis and investigation of isopulegol-based chiral ligands. Int. J. Mol. Sci. 2019, 20, 4050. [CrossRef] [PubMed]

58. Chen, J.; Chen, M.; Zhang, B.; Nie, R.; Huang, A.; Goh, T.W.; Volkov, A.; Zhang, Z.; Ren, Q.; Huang, W. Allylic oxidation of olefins with a manganese-based metal-organic framework. Green Chem. 2019, 21, 3629-3636. [CrossRef]

59. Islam, S.M.; Roy, A.S.; Mondal, P.; Salam, N. Efficient allylic oxidation of olefins catalyzed by polymer supported metal Schiff base complexes with peroxides. J. Inorg. Organomet. Polym. Mater. 2012, 22, 717-730. [CrossRef]

60. Jia, Y.X.; Wu, B.; Li, X.; Ren, S.K.; Tu, Y.Q.; Chan, A.S.C.; Kitching, W. Synthetic studies of the HIV-1 protease ihibitive didemnaketals: Stereocontrolled synthetic approach to the key mother spiroketals. Org. Lett. 2001, 3, 847-849. [CrossRef]

61. Waddell, T.G.; Ross, P.A. Chemistry of 3,4-epoxy alcohols. Fragmentation reactions. J. Org. Chem. 1987, 52, 4802-4804. [CrossRef]

62. Kim, J.H.; Lim, H.J.; Cheon, S.H. A facile synthesis of $\left(6 S, 1^{\prime} S\right)-(+)$-hernandulcin and $\left(6 S, 1^{\prime} R\right.$ )-(+)-epihernandulcin. Tetrahedron 2003, 59, 7501-7507. [CrossRef]

63. Kim, J.H.; Lim, H.J.; Cheon, S.H. Synthesis of (+)-hernandulcin and (+)-epihernandulcin. Tetrahedron Lett. 2002, 43, 4721-4722. [CrossRef]

64. Szakonyi, Z.; Csillag, K.; Fülöp, F. Stereoselective synthesis of carane-based aminodiols as chiral ligands for the catalytic addition of diethylzinc to aldehydes. Tetrahedron Asymmetry 2011, 22, 1021-1027. [CrossRef]

65. Szakonyi, Z.; Csőr, Á.; Csámpai, A.; Fülöp, F. Stereoselective synthesis and modelling-driven optimisation of Carane-based aminodiols and 1,3-oxazines as catalysts for the enantioselective addition of diethylzinc to benzaldehyde. Chem. - Eur. J. 2016, 22, 7163-7173. [CrossRef] [PubMed]

66. Shivani; Pujala, B.; Chakraborti, A.K. Zinc(II) perchlorate hexahydrate catalyzed opening of epoxide ring by amines: Applications to synthesis of $(R S) /(R)$-Propranolols and $(R S) /(R) /(S)$-Naftopidils. J. Org. Chem. 2007, 72, 3713-3722. [CrossRef] [PubMed]

67. Bergmeier, S.C. The synthesis of vicinal amino alcohols. Tetrahedron 2000, 56, 2561-2576. [CrossRef]

68. Gonda, T.; Szakonyi, Z.; Csámpai, A.; Haukka, M.; Fülöp, F. Stereoselective synthesis and application of tridentate aminodiols derived from (+)-pulegone. Tetrahedron Asymmetry 2016, 27, 480-486. [CrossRef]

69. Morikawa, H.; Yamaguchi, J.; Sugimura, S.; Minamoto, M.; Gorou, Y.; Morinaga, H.; Motokucho, S. Systematic synthetic study of four diastereomerically distinct limonene-1,2-diols and their corresponding cyclic carbonates. Beilstein J. Org. Chem. 2019, 15, 130-136. [CrossRef]

70. Tanaka, T.; Yasuda, Y.; Hayashi, M. New chiral Schiff base as a tridentate ligand for catalytic enantioselective addition of diethylzinc to aldehydes. J. Org. Chem. 2006, 71, 7091-7093. [CrossRef]

71. Jimeno, C.; Pastó, M.; Riera, A.; Pericàs, M.A. Modular amino alcohol ligands containing bulky alkyl groups as chiral controllers for Et2Zn addition to aldehydes: Illustration of a design principle. J. Org. Chem. 2003, 68, 3130-3138. [CrossRef]

72. Tashenov, Y.; Daniels, M.; Robeyns, K.; Van Meervelt, L.; Dehaen, W.; Suleimen, Y.; Szakonyi, Z. Stereoselective syntheses and application of chiral bi- and tridentate ligands derived from (+)-Sabinol. Molecules 2018, 23, 771. [CrossRef]

73. Szakonyi, Z.; Hetényi, A.; Fülöp, F. Synthesis and application of monoterpene-based chiral aminodiols. Tetrahedron 2008, 64, 1034-1039. [CrossRef]

74. Yendapally, R.; Lee, R.E. Design, synthesis, and evaluation of novel ethambutol analogues. Bioorg. Med. Chem. Lett. 2008, 18, 1607-1611. [CrossRef] [PubMed]

75. Cunico, W.; Gomes, C.R.B.; Ferreira, M.L.G.; Ferreira, T.G.; Cardinot, D.; de Souza, M.V.N.; Lourenço, M.C.S. Synthesis and anti-mycobacterial activity of novel amino alcohol derivatives. Eur. J. Med. Chem. 2011, 46, 974-978. [CrossRef] [PubMed] 
76. Weinstein, M.P. Methods for Dilution Antimicrobial Susceptibility Tests for Bacteria That Grow Aerobically, 11th ed.; Clinical and Laboratory: Wayne, PA, USA, 2018.

77. Béni, Z.; Dékány, M.; Kovács, B.; Csupor-Löffler, B.; Zomborszki, Z.; Kerekes, E.; Szekeres, A.; Urbán, E.; Hohmann, J.; Ványolós, A. Bioactivity-guided isolation of antimicrobial and antioxidant metabolites from the mushroom Tapinella atrotomentosa. Molecules 2018, 23, 1082. [CrossRef]

Sample Availability: Samples of the compounds 3-15 are available from the authors.

(C) 2019 by the authors. Licensee MDPI, Basel, Switzerland. This article is an open access article distributed under the terms and conditions of the Creative Commons Attribution (CC BY) license (http://creativecommons.org/licenses/by/4.0/). 